\title{
Angular Asymmetry in the Production of Light and Heavy Recoil Nuclides in Proton Induced Reaction with Au Target at GeV Energies
}

\author{
S.K. Sharma* And B. KAMYS \\ Institute of Physics, Jagiellonian University, St. Łojasiewicza 11, 30-348 Kraków, Poland
}

\begin{abstract}
It is shown that model calculations are able to reproduce main properties in terms of experimental mass dependence of the forward-backward asymmetry of the emitted reaction products from proton-Au collisions in the proton beam energy range from $1 \mathrm{GeV}$ to $3 \mathrm{GeV}$. Qualitative as well as quantitative comparisons are done between the measurements and the calculations performed by means of the intra nuclear cascade code INCL4.6 coupled with four different codes: SMM, GEMINI++, ABLA07, and GEM2 with the aim to validate the selected reaction models.
\end{abstract}

DOI: $10.12693 /$ APhysPolA.127.1533

PACS: $25.40 . \mathrm{Sc}, 25.40 . \mathrm{Kv}$

\section{Introduction}

The experimental mass dependence of forwardbackward $(F / B)$ asymmetry of the reaction product emission in proton-Au collisions varies strongly for the proton beam energy range from $1 \mathrm{GeV}$ to $3 \mathrm{GeV}$ [1]. The motivation of current investigations is to study whether the assumed reaction mechanism can reproduce both, the product mass dependence of the $F / B$ asymmetry and its variation with the beam energy. The reaction is treated as a two-step process which consists of the fast stage of the intranuclear cascade of the nucleon-nucleon collisions followed by a slow process of the deexcitation of the target's remnants. The intranuclear cascade model INCL4.6 [2] is used to describe the first step of the process whereas four different models (SMM [3], GEMINI ++ [4], ABLA07 [5] and GEM2 [6]) are applied for the second step. Qualitative as well as quantitative comparisons between the measurements and the theoretical calculations are presented.

The paper is organized as follows: In Sect. 2 we give detailed description on how to calculate the $F / B$ ratio for different nuclides and the qualitative comparison between the model predictions and the experimental data. Section 3 is dedicated to the quantitative analysis which eventually helps to provide the ranking to different models. In Sect. 4, the summary of the results is presented.

\section{The qualitative analysis of $F / B$ asymmetry}

The measurements of the forward/backward asymmetry reported by Kaufman et al. [1], were done for both proton beam energies of $1 \mathrm{GeV}$ and of $3 \mathrm{GeV}$ for a thick $\mathrm{Au}$ target $\left(24 \mathrm{mg} / \mathrm{cm}^{2}\right)$. The comparison of the results

*corresponding author; e-mail: sushil.sharma@uj.edu.pl of model calculations with the data for such a thick target has to be performed taking into consideration the stopping of low energy reaction products in the target.

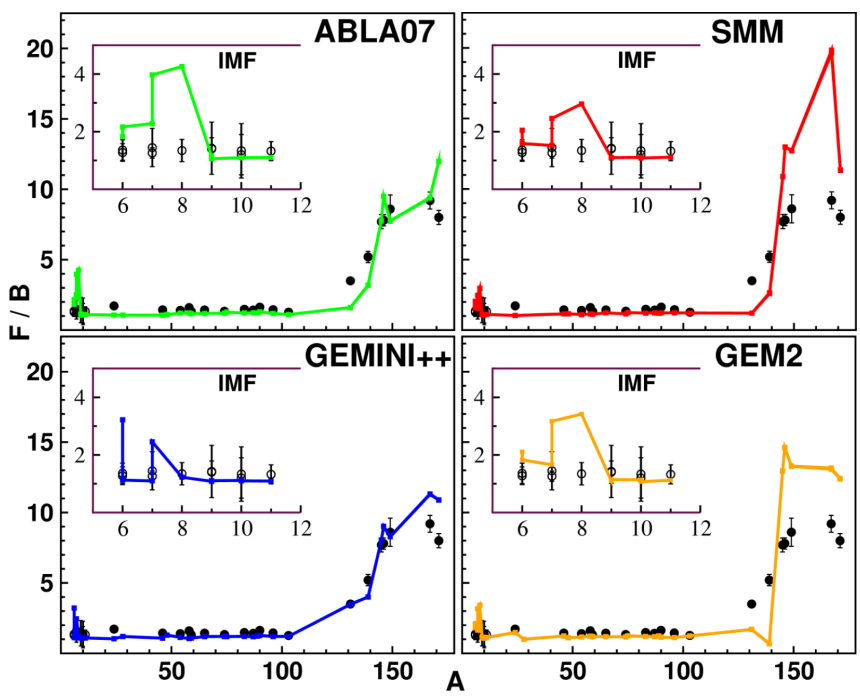

Fig. 1. Comparison between the measurements (points) for the $F / B$ ratio with four different theoretical models (lines): INCL4.6 coupled with ABLA07 (left upper part), SMM (right upper part), GEMINI++ (left lower part), and GEM2 (right lower part), at $1 \mathrm{GeV}$ proton beam energy.

The model calculations of the $F / B$ asymmetry were performed along to the following steps:

(i) Energy loss and the range of different ions in the $\mathrm{Au}$ material were evaluated by using stopping range of ions in matter (SRIM model) for a broad energy range of the ions.

(ii) Random sampling of the interaction point of the proton from the beam with the target nucleus was done in the full thickness of the target since the protons of $\mathrm{GeV}$ 
energies lose only a small part of their energy in the $\mathrm{Au}$ target of the thickness of $24 \mathrm{mg} / \mathrm{cm}^{2}$.

(iii) The fate of all reaction products was simulated event-by-event. The path length of the ion-reaction product from the reaction point to the outer edge of the target was calculated taking into account the direction of its emission. Then this path length was compared with the range of the ion of given energy in the target material.

The reaction products which were able to leave the target were used to calculate the $F / B$ ratio.

The experimental values of the $F / B$ ratio for intermediate mass fragments (IMFs) with the mass number in the range of $6 \leq A \leq 12$ measured at $T_{p}=1.2 \mathrm{GeV}$ [7] and heavy products $(A \geq 24)$ measured at $T_{p}=1.0 \mathrm{GeV}[1]$ for $\mathrm{p}+\mathrm{Au}$ reactions are presented in Fig. 1 (points) whereas the same observable is depicted in Fig. 2 (points) for IMFs at $T_{p}=2.5 \mathrm{GeV}[7]$ and for heavy products at $T_{p}=3.0 \mathrm{GeV}$ [1]. The IMF data are also presented separately in the insets of the figures to show details of the mass dependence of the $F / B$ ratio for these particles.

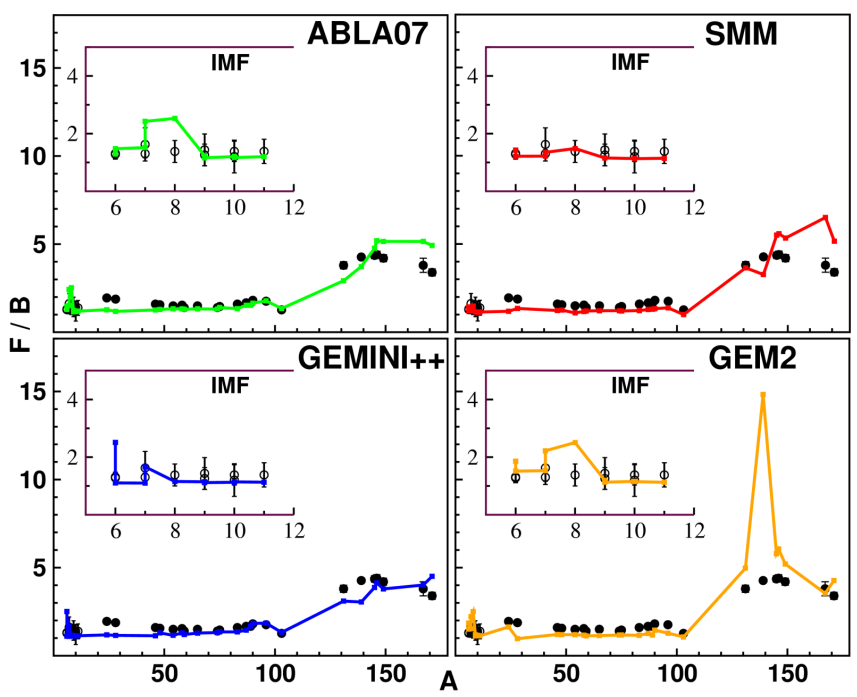

Fig. 2. The same as Fig. 1 but at $3 \mathrm{GeV}$ proton beam energy.

It is clearly visible in Fig. 1 that the shape and magnitude of the mass dependence of the $F / B$ asymmetry is best reproduced by GEMINI ++ (blue line in the left, lower part of the figure) for both IMF and heavy products. Excluding nuclides with masses $A=7$ and 8, IMF are well described by ABLA07, SMM and GEM2. However, the magnitude of the $F / B$ asymmetry of heavy products is significantly overestimated by both, GEM2 and SMM.

The data at higher energy are compared with the model predictions in Fig. 2. The INCL4.6 model coupled with SMM model is the best in reproducing the experimental data for IMFs. It is followed by GEMINI ++ , ABLA07, and GEM2. For heavy products all models are able to reproduce the shape of the mass dependence of $F / B$, whereas GEMINI ++ seems to be successful even in reproducing the magnitude. The GEM2 model overestimates the $F / B$ ratio for the particular fragment with $A=139$.

\section{Ranking of the models}

In order to make judgement about the best models we used quantitative measure of agreement of the data and theoretical $F / B$ ratios. The statistical $H$-test [8] has been used for this purpose

$$
H=\left(\frac{1}{N} \sum_{i=1}^{N}\left(\frac{(F / B)_{i}^{\exp }-(F / B)_{i}^{\mathrm{cal}}}{\Delta(F / B)_{i}^{\exp }}\right)^{2}\right)^{1 / 2} .
$$

Here $(F / B)_{i}^{\exp }$ symbol represents the experimental and $(F / B)_{i}^{\text {cal }}$ the theoretical $F / B$ asymmetry for $i$-th nuclide whereas $\Delta(F / B)_{i}^{\exp }$ is the error of experimental values. The test calculates the difference between the model cross-sections and the experimental values in units of the experimental error. In the case of a perfect agreement the test has value equal to $\approx 1$. More deviation from unity, more the models are in disagreement in reproducing the experimental data. The test values are separately calculated for IMF and for heavy products. To finally conclude the ranking of models the test values are averaged over both beam energies. The results of this procedure are presented in Table.

TABLE I

Ranking of models based on $H$-test averaged over both beam energies.

\begin{tabular}{c|c|c|c|c}
\hline \hline \multirow{2}{*}{ Model } & \multicolumn{2}{|c|}{$\begin{array}{c}\text { (a) Intermediate } \\
\text { mass fragments [7] }\end{array}$} & \multicolumn{2}{c}{$\begin{array}{c}\text { (b) Heavy } \\
\text { nuclides [1] }\end{array}$} \\
\cline { 2 - 5 } & $H$ & Rank & $H$ & Rank \\
\hline ABLA07 & 2.31 & 4 th & 4.74 & 1st \\
GEM2 & 1.94 & 3 rd & 7.18 & 2 nd \\
GEMINI++ & 0.76 & 2nd & 5.02 & 1st \\
SMM & 1.15 & 1st & 6.99 & 2nd
\end{tabular}

\section{Summary}

The INCL4.6 model coupled to GEMINI++, SMM, ABLA07 and GEM2 models is able to reproduce the shape of the mass number dependence of the $F / B$ asymmetry without introducing any free parameters. The model predictions agree also with the beam energy dependence of the $F / B$ asymmetry. Ranking of the models based on the H-test is as follows: for IMF (1) SMM, (2) GEMINI++, (3) GEM2 and (4) ABLA07 and for heavy nuclides: (1) ABLA07, GEMINI++, (2) SMM, GEM2.

\section{Acknowledgments}

One of us (S.K.S.) gratefully acknowledges the support by the Foundation for Polish Science - MPD program, co-financed by the European Union within the European Regional Development Fund. 


\section{References}

[1] S.B. Kaufman, E.P. Steinberg, M.W. Weisfield, Phys. Rev. C 18, 1349 (1978).

[2] A. Boudard, J. Cugnon, J.-C. David, S. Leray, D. Mancusi, Phys. Rev. C 87, 014606 (2013).

[3] J.P. Bondorf, A.S. Botvina, A.S. Iljinov, I.N. Mishustin, K. Sneppen, Phys. Rep. 257, 133 (1995).

[4] D. Mancusi, R.J. Charity, J. Cugnon, Phys. Rev. C 82, $044610(2010)$

[5] A. Kelić, M.V. Ricciardi, K.-H. Schmidt, arXiv:0906.4193, [nucl-th], 2009.
[6] S. Furihata, Nucl. Instrum. Methods Phys. Res. B 171, 251 (2000); S. Furihata, T. Nakamura, J. Nucl. Sci. Technol. 39 Suppl. 2, 758 (2002).

[7] A. Budzanowski, M. Fidelus, D. Filges, F. Goldenbaum, H. Hodde, L. Jarczyk, B. Kamys, M. Kistryn, S. Kistryn, S. Kliczewski, A. Kowalczyk, E. Kozik, P. Kulessa, H. Machner, A. Magiera, B. PiskorIgnatowicz, K. Pysz, Z. Rudy, R. Siudak, M. Wojciechowski, Phys. Rev. C 78, 024603 (2008).

[8] S.K. Sharma, Acta Phys. Pol. B Proc. Suppl. 6, 1161 (2013). 